Short Communication

Genetics of Microorganisms

\title{
Genetic differences among Moraxella bovis and Moraxella bovoculi isolates from infectious bovine keratoconjunctivitis (IBK) outbreaks in southern Brazil
}

\author{
Helena Brocardo Comin ${ }^{1}$ (iD, Robert Domingues ${ }^{2}$, Emanuelle Baldo Gaspar ${ }^{2}$, João Rodrigo Gil De Los \\ Santos $^{3}$ and Fernando Flores Cardoso ${ }^{2,3}$ \\ ${ }^{1}$ Universidade Federal de Pelotas, Programa de Pós-Graduação em Zootecnia, Pelotas, RS, Brazil. \\ ${ }^{2}$ Embrapa Pecuária Sul, Bagé, RS, Brazil. \\ ${ }^{3}$ Universidade Federal de Pelotas, Departamento de Zootecnia, Pelotas, RS, Brazil.
}

\begin{abstract}
The objective of this study was to evaluate the genetic diversity of Moraxella bovis and Moraxella bovoculi bacteria isolated from infectious bovine keratoconjunctivitis (IBK) outbreaks in the state of Rio Grande do Sul, Brazil. The genetic diversity among Moraxella spp. was evaluated by RAPD-PCR, JWP1-JWOPA07-PCR, ERIC-PCR and by sequencing the 16S-23S intergenic regions. Based on the dendrogram, two genetically differentiated clades were observed; 14 isolates were classified as $M$. bovis and 17 as $M$. bovoculi. Genetic distances between the $M$. bovis samples ranged from 0.0379 to 0.4285 , while for $M$. bovoculi the dissimilarities ranged from zero to 0.7297 . Alternatively, based on sequencing analyses of the 16S-23S intergenic region, M. bovis and M. bovoculi isolates were grouped into the same two different clades, but it was not possible to differentiate between isolates within clades. PCR techniques were demonstrated to be a satisfactory tool to unravel the genetic variability among Moraxella spp., while sequencing of the 16S-23S intergenic region was only able to differentiate two species of the Moraxella genus. Despite sampling geographically close regions, we demonstrate considerable genetic diversity in $M$. bovis and $M$. bovoculi strains and genetically distinct $M$. bovis strains co-infecting the same animal.
\end{abstract}

Keywords: Beef cattle, DNA sequencing, genetic diversity, RAPD.

Received: January 16, 2019; Accepted: June 28, 2019.

Infectious bovine keratoconjunctivitis (IBK) is the most important ocular disease in bovine production (Snowder et al., 2005). The main observed symptoms are: intense lacrimation, photophobia, conjunctival swelling, opacity in the center of the cornea and ulceration (Postma et al., 2008). Despite its low mortality, this disease is characterized by high morbidity, as it can affect up to $80 \%$ of the herd (Postma et al., 2008). It causes significant economic and productive losses due to the reduction in weight gain, decrease in milk production, expenses with repetitive treatments, commercial devaluation, and eventual discard of animals that present severe and permanent ocular sequelae (George, 1990; McConnel et al., 2007). There are no estimates of the economic impact of this disease in the last 20 years.

The etiologic agent of IBK is Moraxella bovis, a Gram-negative bacterium, widely disseminated and highly contagious (McConnel et al., 2007). Another Moraxella species, M. bovoculi, has been frequently isolated from oc-

Send correspondence to Helena Brocardo Comin. Universidade Federal de Pelotas, Departamento de Zootecnia, R. Gomes Carneiro 1, 96010-610 Pelotas, RS, Brazil. E-mail: helenacomin.92@ hotmail.com. ular and nasal secretions of animals affected by IBK (Angelos et al., 2007; Sosa and Zunino, 2012; Karthik et al., 2018). However, the role of this species as IBK causal agent is not fully defined, since the experimental infection with this species failed to provoke the disease (Angelos, 2015).

IBK was diagnosed in most Brazilian states and is widely disseminated in the southern region of Rio Grande do Sul state (Turnes, 2001), mainly affecting taurine bovines (Conceição and Turnes, 2003). It is a seasonal disease, with a worldwide distribution and, generally, highly prevalent (McConnel et al., 2007). It affects animals of all ages, but young animals are generally more susceptible (Killinger, 1977).

Therapeutic and prophylactic conduct against IBK includes the use of antimicrobials and vaccines. However, prophylaxis is generally hampered by low vaccine efficacy (O'Connor et al., 2011; Cullen et al., 2017; O'Connor et al., 2019). This situation can be explained by the genetic and antigenic variation between $M$. bovis strains and the presumed presence of other microorganisms involved in this disease (Brown et al., 1998; Kowalski et al., 2017). 
Table 1 - Molecular characteristics of Moraxella spp. isolated from IBK, in five municipalities of Rio Grande do Sul state, Brazil.

\begin{tabular}{|c|c|c|c|c|}
\hline $\mathrm{N}^{\circ}$ & Isolates & Origin & Surge & Homology \\
\hline 1 & $151 \mathrm{OE}$ & Alegrete & 2016 & M. bovis \\
\hline 2 & Taim -2 & Rio Grande & 2016 & M. bovis \\
\hline 3 & $171 \mathrm{OD}$ & Alegrete & 2016 & M. bovis \\
\hline 4 & $171 \mathrm{OE}$ & Alegrete & 2016 & M. bovis \\
\hline 5 & $324 \mathrm{OE}$ & Alegrete & 2016 & M. bovis \\
\hline 6 & $6213 \mathrm{OD}$ & Dom Pedrito & 2016 & M. bovis \\
\hline 7 & 391 OD & Alegrete & 2017 & M. bovis \\
\hline 8 & $391 \mathrm{OE}$ & Alegrete & 2017 & M. bovis \\
\hline 9 & 976 OD & Alegrete & 2016 & M. bovis \\
\hline 10 & $128 \mathrm{OE}$ & Alegrete & 2016 & M. bovis \\
\hline 11 & $6587 \mathrm{ND}$ & Dom Pedrito & 2017 & M. bovis \\
\hline 12 & $6587 \mathrm{OD}$ & Dom Pedrito & 2017 & M. bovis \\
\hline 13 & 6219 OD & Dom Pedrito & 2016 & M. bovis \\
\hline 14 & $6456 \mathrm{OE}$ & Dom Pedrito & 2016 & M. bovis \\
\hline 15 & 666 OD & Alegrete & 2016 & M. bovoculi \\
\hline 16 & Taim - 1 & Rio Grande & 2016 & M. bovoculi \\
\hline 17 & Taim -3 & Santa Vitória do Palmar & 2016 & M. bovoculi \\
\hline 18 & $6052 \mathrm{OE}$ & Dom Pedrito & 2015 & M. bovoculi \\
\hline 19 & $1368 \mathrm{OD}$ & Dom Pedrito & 2016 & M. bovoculi \\
\hline 20 & $1138 \mathrm{OE}$ & Dom Pedrito & 2017 & M. bovoculi \\
\hline 21 & 1192 OD & Dom Pedrito & 2017 & M. bovoculi \\
\hline 22 & $281 \mathrm{OD}$ & Uruguaiana & 2017 & M. bovoculi \\
\hline 23 & $5623 \mathrm{OE}$ & Dom Pedrito & 2015 & M. bovoculi \\
\hline 24 & $330 \mathrm{NE}$ & Alegrete & 2016 & M. bovoculi \\
\hline 25 & $259 \mathrm{OD}$ & Dom Pedrito & 2016 & M. bovoculi \\
\hline 26 & $29 \mathrm{OE}$ & Alegrete & 2016 & M. bovoculi \\
\hline 27 & 1295 OD & Dom Pedrito & 2016 & M. bovoculi \\
\hline 28 & $1362 \mathrm{OE}$ & Dom Pedrito & 2016 & M. bovoculi \\
\hline 29 & $1213 \mathrm{NE}$ & Dom Pedrito & 2017 & M. bovoculi \\
\hline 30 & $120 \mathrm{ND}$ & Uruguaiana & 2015 & M. bovoculi \\
\hline 31 & $120 \mathrm{OD}$ & Uruguaiana & 2015 & M. bovoculi \\
\hline
\end{tabular}

$\mathrm{OD}=$ Right eye $/ \mathrm{OE}=$ Left eye $/ \mathrm{ND}=$ Right nostril and $\mathrm{NE}=$ Left nostril .

The development of molecular techniques allowed the characterization of genetic differences among bacterial isolates. Bacterial genetic variability can be estimated by comparing differently sized DNA fragments, generated from PCR amplification with the use of random primers (Saiki et al., 1988), or by sequencing the internal transcribed spacer (ITS) (Cieslinska et al., 2015; Tokajian et al., 2016).

The main DNA fingerprinting techniques to assess genetic variability among bacteria are PCR-based ones, such as random amplification of polymorphic DNA (RAPD), and repetitive enterobacterial intergenic consensus (ERIC)-PCR. The PCR-based techniques require no specific knowledge of the DNA sequence of the target organism, resulting in several anonymous, not previously de- termined, but reproducibly amplified fragments (Bowditch et al., 1993). RAPD is based on randomic amplification of DNA with decamer primers (Shekhawat et al., 2019). ERIC-PCR is similar to RAPD, but with larger primers (Hulton et al., 1991). These techniques were successfully used to verify the genetic variability between $M$. bovis and M. bovoculi strains in studies focused on the epidemiology of IBK (Prieto et al., 1999; Conceição et al., 2004; Sosa and Zunino, 2013). On the other hand, DNA sequencing of ITS is valuable for species identification (Cieslinska et al., 2015; Tokajian et al., 2016), due to the high variability in this region. Additionally, it can be used to trace inter- and intraspecific genetic variability (Spacov et al., 2006).

The objective of the present study was to characterize the genetic diversity of $M$. bovis and $M$. bovoculi isolates from IBK outbreaks between 2015 and 2017 from five municipalities in Rio Grande do Sul, Brazil, by RAPD-PCR, JWP1-JWOPA07-PCR, ERIC-PCR, and sequencing the ITS.

The study was approved by Ethics Committee on Animal Experimentation of the Federal University of Pelotas (approval number 2328-2017, approved at 02 October 2017). Five non-vaccinated herds, with most of the cows displaying signs of IBK at the time of collection were enrolled in the study. Around 18 animals per herd were sampled, although the number of animals in each herd was 120 on average. Four samples per animal were collected, totalizing 360 samples, from 90 cattle with initial clinical symptoms of IBK. Swabs from both eyes and nostrils were collected, immediately seeded on blood agar, incubated at $37^{\circ} \mathrm{C}$, and $24 \mathrm{~h}$ later, small, white and $\beta$-hemolytic colonies were re-seed on blood agar for further characterization. From 360 samples, 28 Moraxella spp. were isolated and characterized as M. bovis and M. bovoculi by PCR-RFLP, according to Angelos and Ball (2007). Additionally, three other strains, previously isolated and identified as $M$. bovis and M. bovoculi (Taim-1; Taim-2 and Taim-3) were added to the study. All samples were collected in Rio Grande do Sul state, Brazil, totalizing 31 samples (Table 1).

The extraction of genomic DNA was based on the protocol developed by Ausubel et al. (2003). The concentration and purity of DNA samples were evaluated by nano-spectrophotometry (NanoDrop ND-2000), and DNA integrity by $1 \%$ agarose gel electrophoresis.

Initially, the genetic diversity was evaluated by RAPD. For primer selection, a set of 20 decamer primers with arbitrary sequence (OPA-01 to OPA-20 - Operon Technologies, Alameda, USA) was tested with two $M$. bovis and two M. bovoculi samples. The primers that produced a higher number of analyzable amplicons, i.e. producing thick and strong bands, were chosen for the reproducibility of the technique. Amplification reactions used the parameters of Domingues et al. (2011). DNA fragments from PCR amplification were loaded on a 1.5\% agarose gel and submitted to electrophoresis in TBE buffer, stained 
with ethidium bromide, and photo-documented on UV light using the Imaging Systems (UVITEC). This initial step allowed to choose seven primers that were then used to analyze all the samples.

The degree of genetic diversity among Moraxella spp. was also investigated with primers the JWP1JWOPA07 and ERIC, according to Sosa and Zunino (2013), who had used them to assess the genotypic diversity of the isolates of $M$. bovis and $M$. bovoculi, from Kansas (USA) and Uruguay. The primers used for amplification were: JWP1 (5'-GCACTGAAGTGACCAAGCGG-3') and JWOPA7 (5'-GAAACGGGTG-3'), ERIC-2 (5'-AA GTAGTGACTGGGGTGAGCG-3') and ERIC-1R (5'ATGTAAGCTCCTGGGGATTCAC-3') (Sosa and Zunino, 2013). For ERIC-PCR we used amplification conditions slightly different from Sosa and Zunino (2013), including an initial denaturation step at $94{ }^{\circ} \mathrm{C}$ for $5 \mathrm{~min}$, followed by 45 cycles of $1 \mathrm{~min}$ at $94{ }^{\circ} \mathrm{C}, 1 \mathrm{~min}$ at $52{ }^{\circ} \mathrm{C}$ and 2 $\min$ at $72{ }^{\circ} \mathrm{C}$, with a final extension at $72{ }^{\circ} \mathrm{C}$ for $10 \mathrm{~min}$. The products of the reactions were analyzed by agarose gel electrophoresis, as previously explained.

To analyze the amplicons, a matrix with binary data (presence $=1$ or absence $=0$ ) was constructed for the quantification of genetic diversity using the GENES software (Cruz, 2008). Estimates of genetic similarity between each pair of lineages were obtained by means of the Dice similarity coefficient (Reif et al., 2005), observing the interval of occurrence between one (putatively clones) to zero (highly divergent). A dendrogram was constructed using the matrix of dissimilarity estimated by unweighted paired group method of cluster analysis using arithmetic averages (UPGMA) (Cruz and Carneiro, 2003).

For ITS sequencing, after amplification of the $16 \mathrm{~S}$ 23S intergenic regions (Angelos et al., 2007), the amplicons were purified with kit MinElute ${ }^{\circledR}$ (Qiagen $\left.{ }^{\circledR}\right)$. For Sanger sequencing, the reactions were carried out with kit BigDye Terminator v3.1 Cycle Sequencing, following the conditions laid down by the manufacturers. After the reaction, the generated fragments were subjected to capillary electrophoresis in a ABI 3500 Genetic Analyzer (Applied Biosystems). Consensus sequences were generated for each sample in DNA Baser (v.5.15) and aligned with sequences from GenBank (accesses: Moraxella bovis CP030241.1; DQ647927.1; EU014535.1; EU014547.1 and EU014575.1, and Moraxella bovoculi - DQ153085.1; DQ153089.1 and DQ153093.1), and a phylogenetic tree was constructed by the Maximum Likelihood method using MEGA (v7.0.26).

In our study, the bacteria were isolated from around $10 \%$ of the herd, only from ill animals. Bacteria isolation prior to identification allows only viable bacteria to be detected. In addition, we focused only on hemolytic colonies, since the cytotoxin $\beta$-hemolysin is a known virulence factor to $M$. bovis (Postma et al., 2008). We identified 13/28 M. bovis, and 15/28 M. bovoculi, from 90 animals, contra- dicting previous works that more frequently isolated $M$. bovis (O'Connor et al., 2012). In our study, no M. ovis was isolated, while Schnee et al. (2015), who worked with Moraxella identification in pre-IBK, post-IBK, and acute IBK phase herds, observed M. ovis more frequently, but concluded that only $M$. bovoculi was directly related to the disease, since it was the only species with increased prevalence only in the acute IBK phase herd.

Using the PCR-derived techniques, the selected primers for RAPD (OPA-02, OPA-03, OPA-04, OPA-07, OPA-09, OPA-11 and OPA-13 - Table S1), JWP1JWOPA07 and ERIC-PCR (PCR-derived techniques) generated together 107 analyzable amplicons ranging from six (OPA-07) to 19 (OPA-03) (Figure 1). Analyzable amplicons were used to generate a dendrogram (Figure 2) from a unique diversity matrix. Two main genetically differentiable groups (clades) were observed. As expected, the $14 \mathrm{M}$. bovis isolates remained in one clade, whereas the $17 \mathrm{M}$. bovoculi remained in the other (Figure 2). According to the calculated bootstrap values, the two clades have great stability, reaching values of $76.15 \%$ and $92.35 \%$ for $M$. bovis and $M$. bovoculi, respectively.

Genetic distances between $M$. bovis samples ranged from 0.0379 to 0.4285 , with a mean of 0.1970 . The lowest values of genetic dissimilarity were recorded among the 391 OD and 171 OD isolates, collected from two distinct animals, but belonging to the same herd. On the other hand, samples 6213 OD and $391 \mathrm{OE}$ were the genetically most distant, both collected from distinct animals of different municipalities.

The values of dissimilarities between $M$. bovoculi ranged from zero to 0.7297 , with a mean of 0.3625 . Among the samples studied, two are possibly clones (120 OD and $120 \mathrm{ND}$ ), what is not surprising, since they were sampled from the same animal (Figure 2). The highest index of genetic divergence was verified between the samples Taim-3 and $1213 \mathrm{NE}$, these being samples collected from different animals belonging to two farms located in different municipalities. Despite the low number of samples and the geographic proximity between collection areas, genetic diversity analyzed by PCR-derived techniques proved to be a satisfactory tool to reveal the existence of genetic variability/similarity among Moraxella spp. Except for one herd, we always isolated for both, M. bovis and M. bovoculi, strains with different genetic profiles from animals coexisting in the same area (data not shown), corroborating previous work that demonstrated genetic variability of Moraxella isolates within the herds (Conceição et al., 2004).

By means of PCR-derived techniques, several previous studies have demonstrated genetic differences between isolates of M. bovis (Prieto et al., 1999; Conceição et al., 2004) or M. bovis and M. bovoculi (Sosa and Zunino, 2013) in Brazil, Argentina, and Uruguay, regions geographically related to the sampling of our study. The only previous study considering Brazilian isolates visualized a consider- 


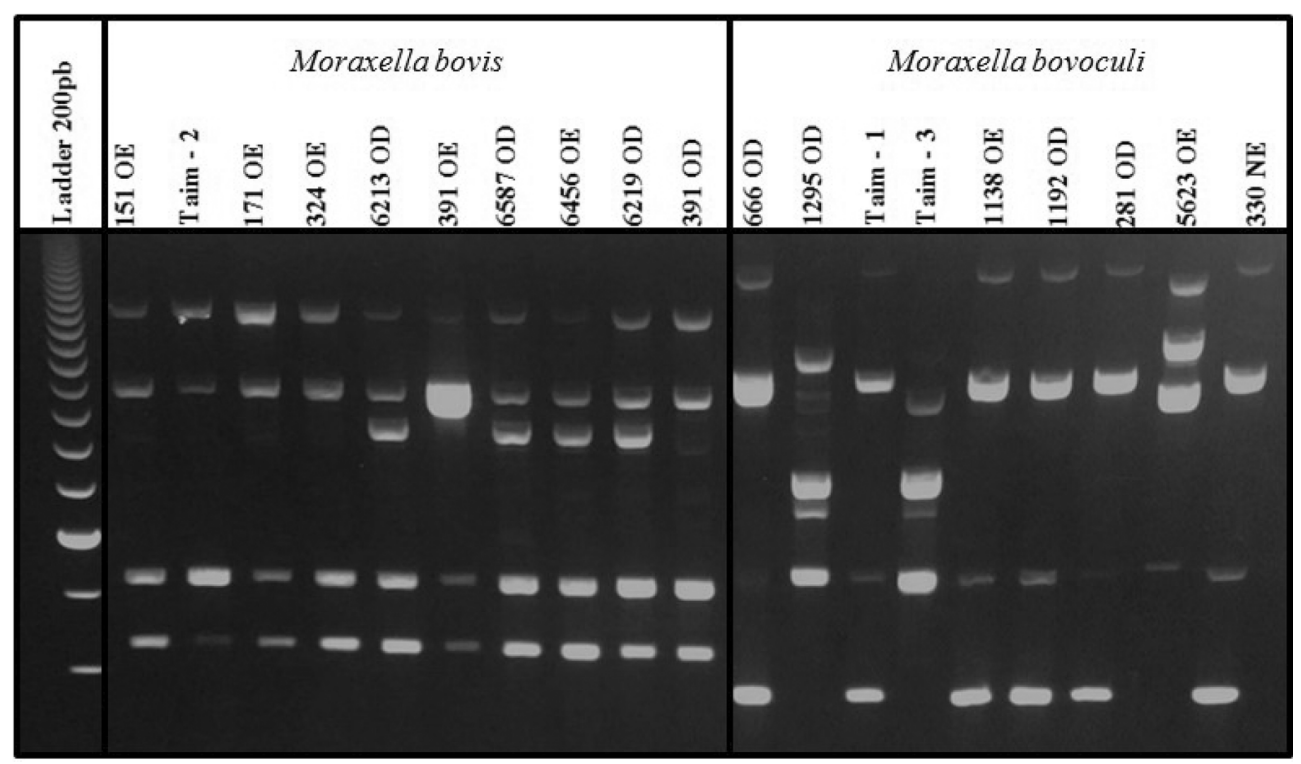

Figure 1 - Example of RAPD technique using a single primer (OPA-03). After RAPD-PCR, ten samples of M. bovis (left side) and nine samples of $M$. bovoculi (right side) were run in an agarose gel and stained with ethidium bromide.

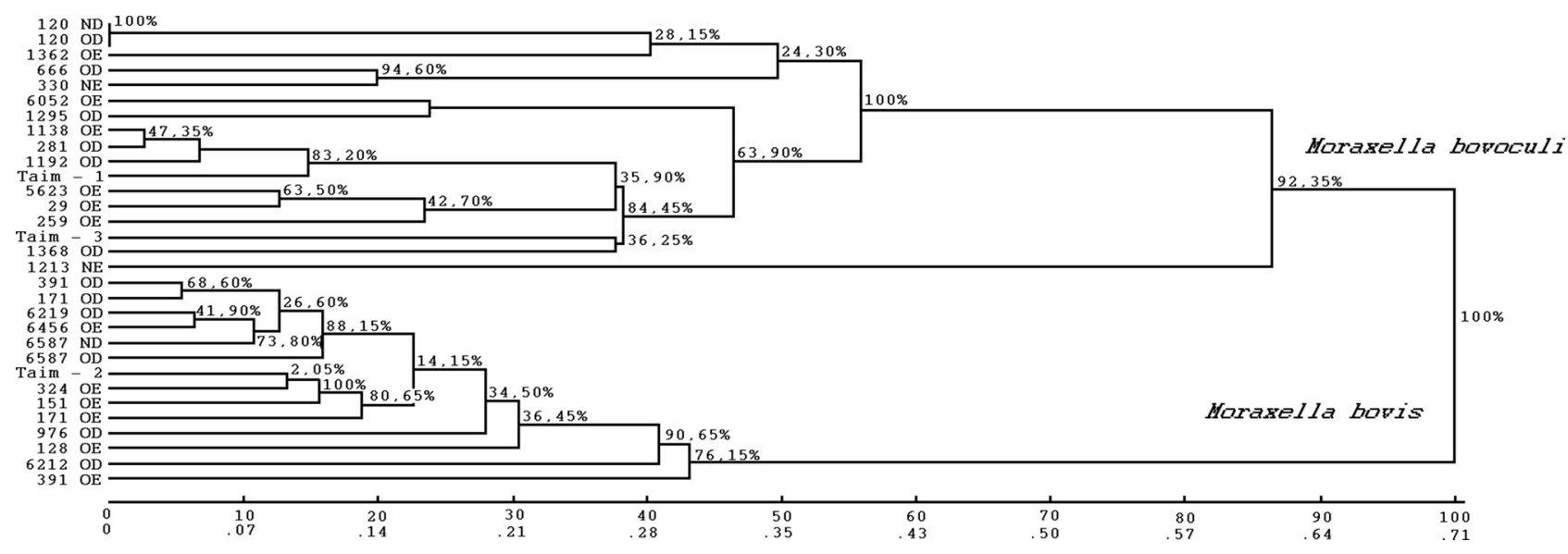

Figure 2 - Dendrogram representing the genetic relationships between M. bovis and M. bovoculi isolates by the UPGMA method. Bootstrap values represented in the tree (2000 bootstrap repetitions).

able higher number of possible clones than we did, possible due to the smaller number of analyzed amplicons (Conceição et al., 2004).

We also constructed a phylogenetic tree based on the homology of the ITS sequence of the Moraxella spp. isolates (Figure 3). M. bovis and M. bovoculi were grouped into two different clades, corroborating with the dendrogram analysis (Figure 2). This technique was able to satisfactorily identify/differentiate the two species of the Moraxella genus; however it was less informative than PCR-derived techniques to reveal genetic differences between isolates. It produced a less branched tree, and the concordance between the different intraspecific clades (Figure 3) with those obtained by PCR techniques (Figure 2), was not comprehensive. This apparent inconsistency, nonetheless, could be due to differences in evolutionary pressure in different parts of genome. ITS sequencing was less effective in demonstrating genetic variation, probably because regions with significant interspecies variations but low intraspecific polymorphisms were sequenced, corroborating a previous study (Wang et al., 2008). The higher discriminatory power of RAPD markers can be explained by the greater coverage of these markers throughout the whole genome. An alternative for increasing the discriminatory power of sequencing is to target additional regions of the bacterial genome within the same analysis.

Interestingly, three $M$. bovis pairs, each one isolated from the same animal (171 OD - $171 \mathrm{OE} ; 391 \mathrm{OD}-391 \mathrm{OE}$ and 6587 OD - 6587 ND) were not genetically identical, when analyzed by PCR-derived techniques. These pairs showed genetics distance of $0.1304 ; 0.3333$ and 0.1034 , respectively (Figure 2). On the other hand, according to ITS 


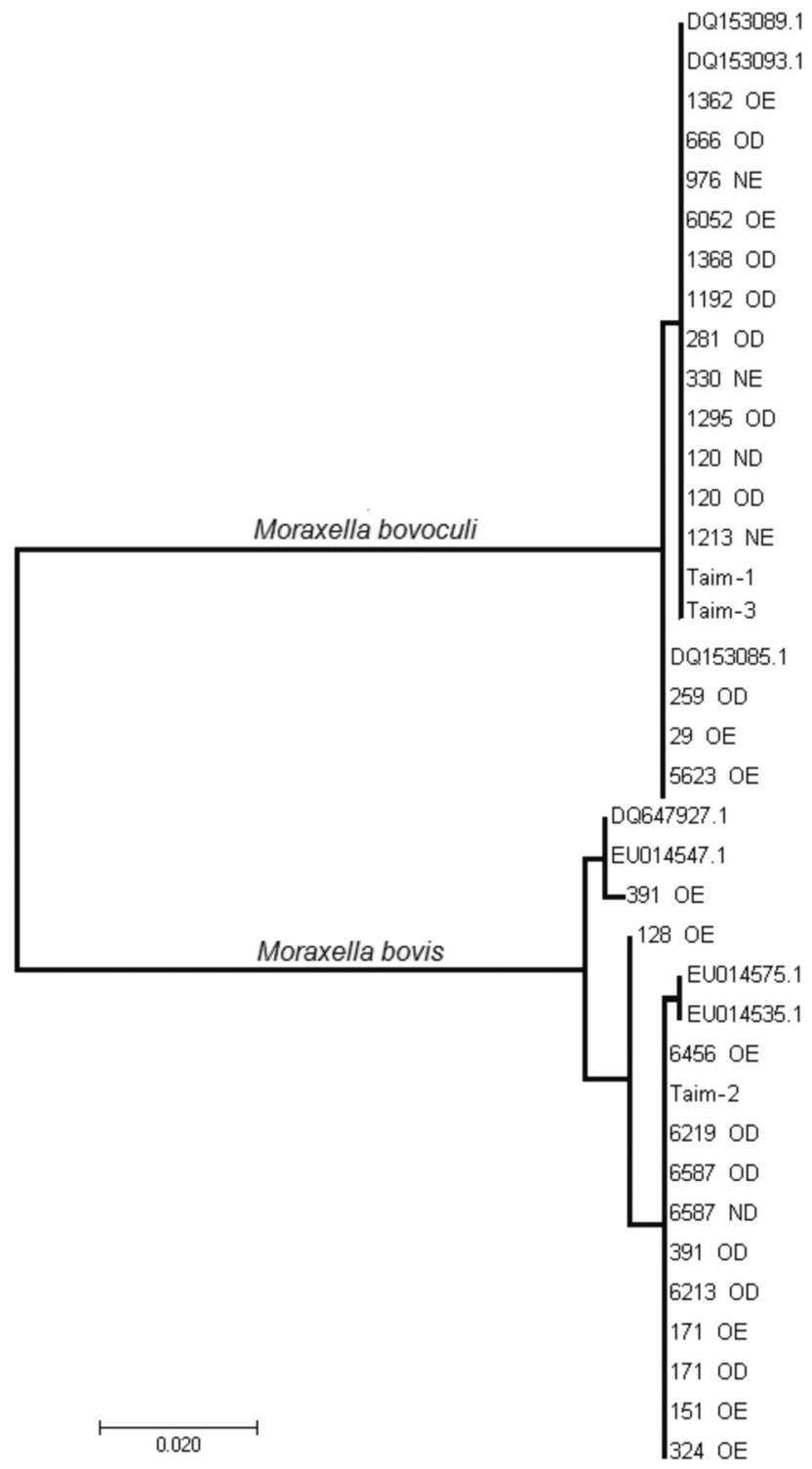

Figure 3 - Phylogenetic tree of the ITS region of the M. bovis and M. bovoculi isolates inferred by the maximum likelihood algorithm.

sequencing, only the pair isolated from animal $391 \mathrm{di}-$ verged at $7 \mathrm{bp}$ of the $656 \mathrm{bp}$ analyzed (1.07\%). This pair presented the highest distance when analyzed by PCRderived techniques $(0.3333)$. Considering only samples from animal 391, the 391 OD was more similar with the others collected in this work and with accesses EU014535.1 and EU014575.1 from GenBank, whereas the 391 OE sample was more similar with the GenBank samples EU014547.1 and DQ647927.1 (Figure 3). Consequently, samples 391 OD and 391 OE stayed in separate clades.

The co-infection of single animals with genetically distinct strains has not yet been documented for M. bovis, although for other bacteria this phenomenon was described (Ogle and Vasil, 1991; Gaspar et al., 2011). This can be a complicating factor in the treatment of infections and in the efficacy of vaccines (Brown et al., 1998; Cullen et al.,
2017), because different strains may have different antigenic and genetic properties, as well as distinct susceptibility profiles to antimicrobial (Gaspar et al., 2011). We previously tested the antimicrobials susceptibility in these isolates and found no difference, however (Comin et al., 2017)

Although there is circumstantial evidence of $M$. bovoculi involvement in the pathogenesis of IBK, it has not yet been possible to prove the participation in the etiology of the disease (Gould et al., 2013). In our study, M. bovoculi was frequently isolated from animals with clinical cases, corroborating several previous studies (Angelos et al., 2010; Libardoni et al., 2012; Karthik et al., 2018), and in some cases it was the only Moraxella species isolated from the herd. We also demonstrated a higher genetic difference for M. bovoculi, in comparison to M. bovis, which agrees with the recent findings that demonstrate, by sequencing, high genetic variability in this species (Dickey et al., 2016).

Even with those evidences, currently the only commercial vaccine in Brazil includes only one strain of $M$. bovoculi among the antigens, while in the US, the vaccine contains eight serotypes. Studies showing correlation between genetic and antigenic variability are scarce. Conceição et al. (2004) showed low correspondence between these two parameters, for M. bovis. However, most recent studies involving the sequencing of cytotoxin from $M$. bovis, $M$. bovoculi, and $M$ ovis showed genetic variability in the aminoacid sequence for this virulence factor, depending on the period of isolation (Farias et al., 2015). Moreover, for M. catharralis, the head domains of UspA2/2H promotes a general evasion of the host immune system throughout the extensive sequence polymorphism in this protein (Su et al., 2013). However, these two works did not correlate those differences with serological variability, nor with genetic diversity. Taken together, these findings suggest a possible variability in antigenic composition, in addition to genetic variation. Thus, we postulate that the high genetic variability can be translated as antigenic variability, hampering the universality of vaccines.

Despite sampling geographically close regions, we demonstrated considerable genetic diversity in $M$. bovis and $M$. bovoculi strains, indicating that the species must present a corresponding antigenic diversity, which can negatively affect therapeutic choice, control measures, and mainly, vaccine efficiency. Moreover, we demonstrated for the first time that $M$. bovis genetically distinct strains can co-infecting the same animal, which can limit therapeutic and vaccine efficiency, even within a single farm.

\section{Acknowledgments}

This research was funded by Embrapa (project number 02.13.10.002.00). The authors acknowledge the contribution of Hereford breeders: Paulo Fleck, Valter José Pötter, Patrícia Wolf and Eduardo Einchenberg. The authors 
also thank funding agencies CAPES (Finance code 001) and CNPq, as well as the Federal University of Pelotas.

\section{Conflict of Interest}

The authors declare that there is no conflict of interest associated with this publication.

\section{Author Contributions}

HBC RD EBG and FFC: Conceptualization, HBC RD and EBG: Formal analysis, EBG RD and FFC: Funding acquisition, HBC RD EBG JRGS and FFC: Investigation, HBC RD EBG and JRGS: Methodology, EBG and FFC: Project administration, EBG RD and FFC: Supervision, HBC: Writing - original draft, RD EBG JRGS and FFC: Writing - reviewing and editing

\section{References}

Angelos JA (2015) Infectious bovine keratoconjunctivitis (Pinkeye). Vet Clin Food Anim Pract 31:61-79.

Angelos JA and Ball LM (2007) Differentiation of Moraxella Bovoculi sp. nov. from other coccoid Moraxellae by the use of polymerase chain reaction and restriction endonuclease analysis of amplified DNA. J Vet Diagn Invest 19:532-534.

Angelos JA, Spinks PQ, Ball LM and George LW (2007) Moraxella bovoculi $\mathrm{sp}$. nov., isolated from calves with infectious bovine keratoconjunctivitis. Int J Syst Evol Microbiol 57:789-795.

Angelos JA, Lane VM, Ball LM and Hess JF (2010) Recombinant Moraxella bovoculi cytotoxin-ISCOM matrix adjuvanted vaccine to prevent naturally occurring infectious bovine keratoconjunctivitis. Vet Res Commun 34:229-239.

Ausubel FM, Brent R, Kingston RE, Moore DD, Seidman JG, Smith JA and Struhl K (2003) Current protocols in molecular biology. Wiley, New York, 4755 p.

Bowditch BM, Albright DG, Williams JGK and Braun MJ (1993) Use of randomly amplified polymorphic DNA markers in comparative genome studies. Meth Enzymol 224:294-309.

Brown MH, Brightman AH, Fenwick BW and Rider MA (1998) Infectious bovine keratoconjunctivitis: A review. J Vet Intern Med 12:259-266.

Cieslinska M, Hennig E, Kruczynska D and Bertaccini A (2015) Genetic diversity of 'Candidatus Phytoplasma mali' strains in Poland. Phytopathol Mediterr 54:477-487.

Comin HB, Gaspar EB, Domingues R, Gil de Los Santos JR, Schramm R and Cardoso FF (2017) Susceptibilidade de cepas de Moraxella bovis e Moraxella bovoculi frente aos antimicrobianos. In: XIX Encontro de Pós-Graduação (ENPOS), Pelotas.

Conceição FR and Turnes CG (2003) Moraxella bovis: influência das características genotípicas e fenotípicas no controle da Ceratoconjuntivite Infecciosa Bovina. Cienc. Rural 33:778-787.

Conceição FR, Dellagostin OA, Paolichi F, Leturia AC and Turnes CG (2004) Molecular diversity of Moraxella bovis isolated from Brazil, Argentina and Uruguay over a period of three decades. Vet J 167:53-58.

Cruz CD (2008) Programa Genes: Diversidade Genética. Editora UFV, Viçosa, 278 pp.
Cruz CD and Carneiro PCS (2003) Modelos biométricos aplicados ao melhoramento genético. 3rd edition. Editora UFV, Viçosa, 623p.

Cullen JN, Engelken TJ, Cooper V and O'Connor AM (2017) Randomized blinded controlled trial to assess the association between a commercial vaccine against Moraxella bovis and the cumulative incidence of infectious bovine keratoconjunctivitis in beef calves. J Am Vet Med Assoc 251:345-351.

Dickey AM, Loy JD, Bono JL, Smith TPL, Apley MD, Lubbers BV, DeDonder KD, Capik SF, Larson RL, White BJ et al. (2016). Large genomic differences between Moraxella bovoculi isolates acquired from the eyes of cattle with infectious bovine keratoconjunctivitis versus the deep nasopharynx of asymptomatic cattle. Vet Res 47:31.

Domingues R, Machado MA, Forzza RC, Melo TD, WohlresViana S and Viccini LF (2011) Genetic variability of an endangered Bromeliaceae species (Pitcairnia albiflos) from the Brazilian Atlantic rainforest. Genet Mol Res 10:2482-2491.

Farias LD, Maboni G, Matter LB, Scherer CFC, Libardoni F and Vargas AC (2015) Phylogenetic analysis and genetic diversity of 3' region of rtxA gene from geographically diverse strains of Moraxella bovis, Moraxella bovoculi and Moraxella ovis. Vet Microbiol 178:283-287.

George LW (1990) Antibiotic treatment of infectious bovine keratoconjunctivitis. Cornell Vet 80:229-235.

Gaspar EB, Neves PR, Levy CE, Mamizuka EM and Lincopan N (2011) Genetic heterogeneity of carbapenem-resistant Pseudomonas aeruginosa isolates co-infecting the cerebrospinal fluid of a pediatric patient. Diagn Microbiol Infect Dis 70:568-570.

Gould S, Dewell R, Tofflemire K, Whitley RD, Millman ST, Opriessnig T and O'Connor AM (2013) Randomized blinded challenge study to assess association between Moraxella bovoculi and infectious bovine keratoconjunctivitis in dairy calves. Vet Microbiol 164:108-115.

Hulton CSJ, Higgins CF and Sharp PM (1991) ERIC sequences: A novel family of repetitive elements in the genomes of Escherichia coli, Salmonella typhimurium and other enterobacteria. Mol Microbiol 5:825-834.

Karthik K, Mahaprabhu R, Roy P and Raman M (2018) Emergence of Moraxella bovoculi associated with keratoconjunctivitis in an organized dairy farm of India. Proc Nat Acad Sci India Sect B 88:1409-1412.

Killinger AH, Valentim D, Mansfield ME, Ricketts GE, CMArik GF, Neumann AH and Norton HW (1977) Economic impact of infectious bovine keratoconjunctivitis in beef calves. Vet Med Small Anim Clinician 72:618-620.

Kowalski AP, Maboni G, Gressler LT, Espíndola JP, Balzan C, Tasca C, Guizzo JA, Conceição FR, Frandoloso R and Vargas AC (2017) Antigenic characterization of Moraxella bovis, Moraxella bovoculi and Moraxella ovis strains with potential use in vaccines. Vet Microbiol 210:56-63.

Libardoni F, Scherer CFC, Farias L, Vielmo A, Balzan C and Vargas AC (2012) Moraxella bovoculi em casos de ceratoconjuntivite infecciosa bovina no Rio Grande do Sul. Pesq Vet Bras 32:743-746.

McConnel C, Shum L and House J (2007) Antimicrobial susceptibility of Australian bovine Moraxella isolates. Aust Vet J 85:70-71. 
O'Connor AM, Brace S, Gould S, Dewell R and Engelken T (2011) A randomized clinical trial evaluating a farm-oforigin autogenous Moraxella bovis vaccine to control infectious bovine keratoconjunctivis (pinkeye) in beef cattle. J Vet Intern Med 25:1447-1453.

O'Connor AM, Shen HG, Wang C and Opriessnig T (2012) Descriptive epidemiology of Moraxella bovis, Moraxella bovoculi and Moraxella ovis in beef calves with naturally occurring infectious bovine keratoconjunctivitis (pinkeye). Vet Microbiol 155:374-380.

O'Connor A, Cooper V, Censi L, Meyer E, Kneipp M and Dewell G (2019) A 2-year randomized blinded controlled trial of a conditionally licensed Moraxella bovoculi vaccine to aid in prevention of infectious bovine keratoconjunctivitis in Angus beef calves. J Vet Intern Med 33:2786-2793.

Ogle JW and Vasil ML (1991) Genetic heterogeneity in strains of Pseudomonas aeruginosa from patients with cystic fibrosis. J Clin Microbiol 29:663-665.

Postma GC, Carfagnini JC and Minatel L (2008) Moraxella bovis pathogenicity: An update. Comp Immunol Microbiol Infect Dis 31:449-458.

Prieto CI, Aguilar OM and Yantorno OM (1999) Analyses of lipopolysaccharides, outer membrane proteins and DNA fingerprints reveal intraspecies diversity in Moraxella bovis isolated in Argentina. Vet Microbiol 70:213-223.

Reif JC, Melchinger AE and Frisch M (2005) Genetical and mathematical properties of similarity and dissimilarity coefficients applied in plant breeding and seed bank management. Crop Sci 45:1.

Saiki RK, Gelfand DH, Stoffel S, Scharf SJ, Higuchi R, Horn GT, Mullis KB and Erlich HA (1988) Primer directed enzymatic amplification DNA with a thermostable DNA polymerase. Science 239:487491.

Schnee C, Heller M, Schubert E and Sachse K (2015) Point prevalence of infection with Mycoplasma bovoculi and Moraxella spp. in cattle at different stages of infectious bovine keratoconjunctivitis. Vet J 203:92-96.

Shekhawat SS, Gaurav A, Joseph B, Kumar H and Kumar N (2019) Random amplified polymorphic DNA-based molec- ular heterogeneity analysis of Salmonella enterica isolates from foods of animal origin. Vet World 12:146-154.

Snowder GD, Van Vleck LD, Cundiff LV and Bennett GL (2005) Genetic and environmental factors associated with incidence of infectious bovine keratoconjunctivitis in preweaned beef calves. J Anim Sci 83:507-518.

Sosa V and Zunino P (2012). Molecular and phenotypic analysis of Moraxella spp. associated to infectious bovine keratoconjunctivitis in Uruguay. Vet J 193:595-597.

Sosa V and Zunino P (2013) Diversity of Moraxella spp. strains recovered from infectious bovine keratoconjunctivitis cases in Uruguay. J Infect Dev Ctries 7:819-824.

Spacov ICG, Silva SAM, Júnior MAM and Morais MMC (2006) Polymorphism of the rDNA and tDNA loci in clinical isolates of Pseudomonas aeruginosa: a perspective for molecular epidemiology surveillance. Genet Mol Biol 29:722-729.

Su YC, Hallström BM, Bernhard S, Singh B and Riesbeck K (2013) Impact of sequence diversity in the Moraxella catarrhalis UspA2/UspA2H head domain on vitronectin binding and antigenic variation. Microbes infect 15:375-387.

Tokajian S, Issa N, Salloum T, Ibrahim J and Farah M (2016) 16S-23S rRNA gene intergenic spacer region variability helps resolve closely related sphingomonads. Front Microbiol 7:149.

Turnes CG (2001) Ceratoconjuntivite bovina infecciosa. In: RietCorrea F, Schild AL, Mendez MC and Lemos RA (eds) Doenças de Ruminantes e Equinos. Livraria Varela, São Paulo, 426 pp.

Wang M, Cao B, Yu Q, Liu L, Gao Q, Wang L and Feng L (2008) Analysis of the 16S-23S rRNA gene internal transcribed spacer region in Klebsiella species. J Clin Microbiol 46:3555-3563.

\section{Supplementary material}

The following online material is available for this article: Table S1 - RAPD primers.

Associate Editor: Célia Maria de Almeida Soares

License information: This is an open-access article distributed under the terms of the Creative Commons Attribution License (type CC-BY), which permits unrestricted use, distribution and reproduction in any medium, provided the original article is properly cited. 